AIAA-2002-4275

\title{
Ionization non-equilibrium and ablation phenomena in a micro- Pulsed Plasma Thruster
}

\author{
M. Keidar* and I. D. Boyd*
}

Department of Aerospace Engineering, University of Michigan, Ann Arbor, MI 48109, USA.

\begin{abstract}
In this paper, plasma-surface interactions are considered in a micro-Pulsed Plasma Thruster (micro-PPT). Two inter-related problems are addressed here, namely propellant ablation and formation of the ionization layer. The ablation mode is determined by the conditions of the plasma flow outside of the Knudsen layer. We found smooth transition between two ablation modes with small velocity at the edge of the Knudsen layer and limited (sound) velocity at the Knudsen layer edge. In the first case the fraction of backflux is very large, while in the second case it is about $18 \%$ of the primary flux. It is concluded that the transition between these two modes occurs as current density increases. A non-equilibrium ionization model is also developed. It is shown that both equilibrium and nonequilibrium ionization can be realized in a micro-PPT dependent on the discharge parameters. Based on the nonequilibrium ionization model, plasma generation and near-plume expansion is calculated for a micro-PPT. Good agreement with experimental measurements of the electron and neutral densities is found.
\end{abstract}

\footnotetext{
- Research Scientist, Department of Aerospace Engineering, Member of AIAA

* Associate Professor, Department of Aerospace Engineering, Senior Member of AIAA
} 


\section{Introduction}

Since the early 1960's, pulsed plasma thrusters (PPT's) have become one of the electric propulsion devices considered for various space applications. This concept was among the first accepted for space flight mainly due to their simplicity and hence high reliability ${ }^{1}$. However, it was recognized that the PPT has an efficiency at the low level of $10 \%$ (Ref. 2) and therefore several ways for improvement have been suggested ${ }^{3}$. Currently, PPT's are considered as an attractive propulsion option for stationkeeping and drag makeup purposes of mass and power limited satellites. ${ }^{4}$ Recently, a miniature version of the PPT, the micro-PPT, has being designed at the Air Force Research Laboratory for delivery of very small impulse bit $^{5}$. This is a simplified, miniaturized version of a conventional PPT with a thrust in the $10 \mu \mathrm{N}$ range designed to provide attitude control and stationkeeping for microsatellites.

It was recognized that complete assessment of spacecraft integration effects requires characterization of the plasma plume exhaust of a PPT. There were several attempts in the past to develop a plume model ${ }^{6}$. Recently, a self-consistent, end-to-end model of the PPT and its plume with application to electrothermal ${ }^{7,8}$ and electromagnetic PPT' ${ }^{9}$ was developed. With these models, it became clear that the plasma distribution in the plume field heavily depends upon the upstream boundary conditions. Therefore, the model of plasma generation in these devices becomes a very important aspect of accurate plasma plume simulation.

Inspection of the micro-PPT propellant surface after firing indicated signs of charring and preferential ablation near the electrodes. ${ }^{5,10}$ Our previous model suggests that charring phenomena is associated with flux of returned ions to the surface ${ }^{11}$. Furthermore it was shown that the flux of returned particles depends upon the plasma flow conditions in the Knudsen layer. Therefore, the problem of the Knudsen layer under different conditions during the discharge is of great importance. In addition it should be noted that the flow conditions at the Knudsen layer edge determine the ablation rate, which is one of the key performance parameters of any PPT. On the other hand, it was obtained that significant neutral flux is generated during the main discharge in micro-PPT ${ }^{12}$, which is not related to the late ablation. Plasma composition estimations based on the equilibrium consideration for typical conditions in a micro-PPT lead to the conclusion that the ionization degree should be high. To study these issues, we made detailed analyses of plasma generation under non-equilibrium conditions. 
In the present paper we will focus on several aspects of plasma generation in the Micro-PPT. There are two inter-related problems that we address in this article, namely the plasma generation phenomenon (ablation) and formation of the ionization layer. The first problem is interesting from the standpoint of smooth transition between the two ablation modes with small (subsonic) and sonic condition at the edge of the Knudsen layer. It was shown recently that plasma conditions near the Knudsen layer edge strongly affect ablation. The effect consists in the flux of returned particles to the surface that is determined by the plasma density and temperature in the plasma bulk ${ }^{13}$. In principle, two limits are possible. When the plasma is very dense, the flux of returned particles is large. As a result, the velocity at the edge of the Knudsen layer is small. The opposite limit corresponds to the case when the plasma density is small. The plasma density and temperature depend on the flow conditions in the plasma bulk. One can expect that there is a smooth continuous transition between these two regimes. Another issue under consideration is non-equilibrium ionization. Considering the second problem, we study plasma generation by relaxing an assumption about equilibrium ionization in the propellant surface vicinity.

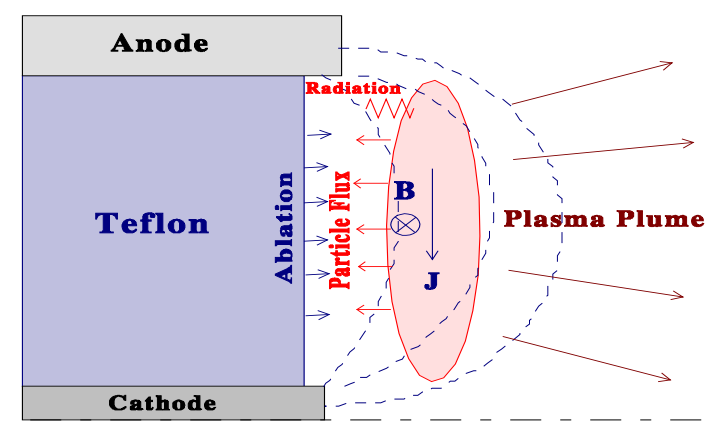

Figure 1. Schematic of the plasma-surface interaction in a micro-Pulsed Plasma Thruster

\section{Ablation phenomena and the Knudsen layer}

There are different characteristic subregions near the surface namely the space-charge sheath, the Knudsen layer, the presheath and a layer where transition to ionization equilibrium occurs, or the socalled ionization layer. These subregions constitute the entire transition layer between a plasma and a wall.

In this section, the problem of evaporation under the condition of strong plasma acceleration near the ablated surface is studied. Similarly to the previous works, we consider the multi-layer structure of the 
near surface region (see Fig. 2). One can distinguish two different characteristic layers between the surface and the plasma bulk: (1) a kinetic non-equilibrium layer adjacent to the surface with a thickness of a few mean free paths (the Knudsen layer), (2) a collision-dominated (hydrodynamic) layer. The definition for the edge of the Knudsen and hydrodynamic layers is considered below.

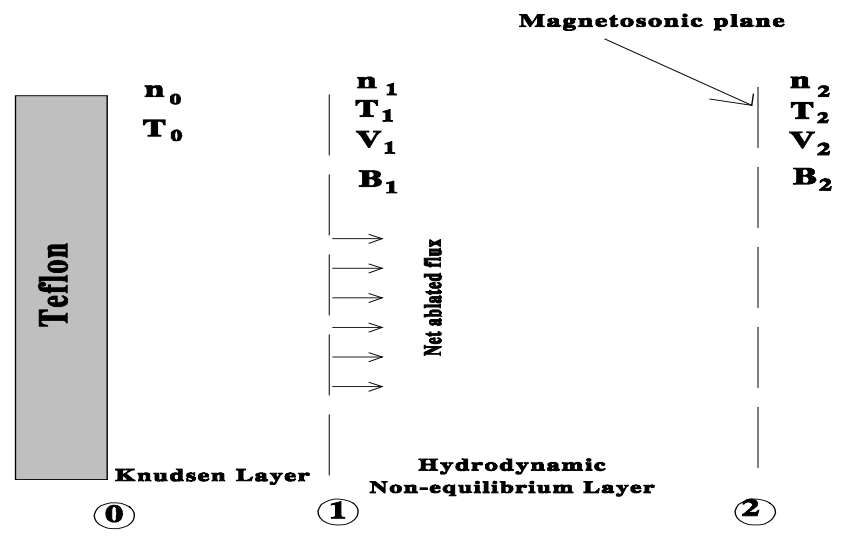

Figure 2. Schematic of the near surface layers

Our formulation starts from the equation set for the hydrodynamic layer. To simplify the present analysis, we assume that ionization equilibrium is reached near the edge of the second layer between boundaries 1 and 2. Analysis of the ionization phenomena is presented in the next section in detail. Therefore, the electron density at the boundary 2 will be calculated using Saha equilibrium. Solution of the hydrodynamic layer problem depends on the boundary conditions at the boundary 1, which is the Knudsen layer edge. To find the parameters at the edge of the Knudsen layer as a function of velocity at the Knudsen layer edge, $\mathrm{V}_{1}$, we apply the mass, momentum and energy conservation equations in kinetic form. Corresponding relations between parameters at the edge of the Knudsen layer and those at the surface were presented elsewhere ${ }^{14}$.

In the hydrodynamic layer, we apply the conservation laws for mass, momentum and energy. Firstly we consider a simplified one fluid MHD description. The magnetic field considered in the model is the selfmagnetic field that is generated as a result of the current between the electrodes. The magnetic field has a primary component in the $y$ direction as shown in Fig. 1. As a result of the magnetic diffusion in the axial direction, the magnetic field decreases between boundaries 1 and 2. The mass conservation equation for heavy particles is: 
$d(n V)=0$

The momentum conservation equation for the plasma is

$M(n V) \frac{d V}{d x}=-\frac{d(n k T)}{d x}+j \times B$

Current density can be calculated from Faraday's law:

$j=\frac{1}{\mu} \cdot \frac{d B}{d x}$

Integration of Eqs. 1-3 yields the following relations between parameters at the boundaries 1 and 2:

$$
n_{1} k T_{1}+M n_{1} V_{1}^{2}+\frac{B_{1}^{2}}{\mu}=n_{2} k T_{2}+M n_{2} V_{2}^{2}+\frac{B_{2}^{2}}{\mu}
$$

The plasma density and velocity distribution in the hydrodynamic layer depend on the specific geometry of the accelerator. Let us consider a planar geometry as an example. Further analysis will be based on the additional assumption about the acceleration condition in the electromagnetic thruster. Using the MACH2 computer code, the existence of a magnetosonic condition at the thruster exit plane was demonstrated. ${ }^{15}$ This assumption was also used in a simplified analytical model ${ }^{16}$ and was adopted in a two-stream performance model of the PPT. ${ }^{17}$ The assumption about a magnetosonic point allows us to reduce the number of unknowns in the problem. Therefore, without missing the main physics, we adopt this assumption. In other words, this assumption is equivalent to the condition that most of the plasma acceleration takes place in the region between boundaries 1 and 2. The Alfven velocity is given by

$$
V_{2}=\frac{B_{2}}{\left(\mu n_{2} M\right)^{0.5}}
$$

To close the system of equations for the hydrodynamic layer, we estimate the magnetic field at the edge of the Knudsen layer as: 
$\mathrm{B}_{1}=\mu \mathrm{jd}$

where $d$ is the characteristic length of the channel (inter-electrode distance) and $j$ is the current density. Combination of the last two equations and Eq. 4 yields the following expression for the velocity at the outer boundary of the kinetic (Knudsen) layer:

$\frac{M V_{1}^{2}}{2 k T_{1}}=\frac{\frac{n_{1}}{2}-\frac{T_{2} n_{2}}{2 T_{1}}+\frac{1}{4} \cdot \frac{\mu(j d)^{2}}{k T_{1}}}{\frac{3}{2} \cdot \frac{n_{1}^{2}}{n_{2}}-n_{1}}$

The calculation of the velocity at the Knudsen layer edge is shown in Fig. 3 as a function of current density. Parameters at the Knudsen layer edge (boundary 1) are calculated using Knudsen layer relations (Ref. 14). Heavy particle density distribution in the acceleration region depends upon the specific geometry of thruster. In order to study only general trends without details of the flow field in this region we consider the total heavy particle density at the magnetosonic plane (boundary 2, Fig. 2) as a parameter of the problem.

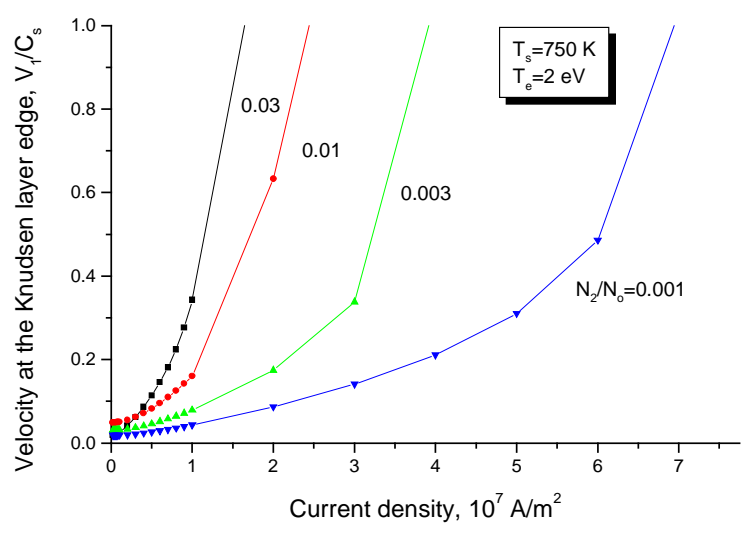

Figure 3. Velocity at the edge of the Knudsen layer as a function of current density with density at the edge of hydrodynamic layer as a parameter. 
One can see that the solution with a small current density corresponds to that for a small velocity in comparison to the sound speed. It should be noted that this solution is close to that obtained in the case of an electrothermal plasma accelerator ${ }^{8}$ and corresponds to ablation into a dense background plasma ${ }^{13}$. Depending on the plasma density at plane 2, the velocity $V_{1}$ increases with current density and approaches to the sound speed limit. The electromagnetic force in the region between boundaries 1 and 2 is responsible for the significant plasma acceleration that in turn affects the Knudsen layer edge. At some value of current density, the solution approaches the limit of the local sound speed. It is interesting to note that no solution beyond the case when velocity $V_{1}$ is equal to the sound speed, since there is no physical mechanism for supersonic acceleration in the Knudsen layer. Therefore density $n_{2}$ when $V_{1}=C_{s}$ can be considered as a limited possible density under the considered conditions. This means that a combination of current density and geometry (that determine the flow field, i.e. $\mathrm{n}_{2}$ ) uniquely determine the solution.

These results show that significant plasma acceleration under external forces (i.e. electromagnetic acceleration in the considered case) affect the boundary conditions at the edge of the Knudsen layer and cause transition from the ablation mode with significant backflux to the ablation mode close to the vacuum case. It should be noted also that our model predicts a smooth transition between these two ablation modes dependent on the current density. 


\section{Ionization layer in the presence of a magnetic field}

In this section we describe the model of the ionization layer for the case of plasma acceleration by an electromagnetic force. The ionization layer can be determined as follows. At the beginning of the ionization layer, the charged particle density is very small and therefore ionization due to particle collisions becomes the primary process. As the charged particle density grows the recombination rate increases and near the edge of the layer the recombination rate becomes close to that of ionization thus establishing equilibrium. Therefore, in reality, the ionization layer edge is determined by recombination. A simplified way to attack the problem is to consider the ionization layer edge as a region where fully ionized plasma is established. Another way to look at this is to consider that a high plasma density and a large electron temperature will lead to establishing a fully ionized plasma. Therefore the ionization layer is the region where transition to a fully ionized plasma occurs.

We start our consideration from the Knudsen layer edge. The reason for this is that in the plasmas we are considering, the mean free path is much larger than the Debye length and therefore the sheath thickness is much smaller than that of the Knudsen layer. Such a scaling analysis allows us to choose proper boundary conditions. We use a zero density boundary condition instead of the Bohm velocity since our starting plane is not at the sheath edge The initial system of equations reads:

$\frac{d\left(n_{i} V_{i}\right)}{d x}=\alpha_{i} n_{i} n_{a}$

$\frac{d\left(n_{i} V_{i}+n_{a} V_{a}\right)}{d x}=0$

$M n_{i} V_{i} \frac{d V_{i}}{d x}=-\frac{d\left(P_{i}+P_{e}\right)}{d x}-\frac{1}{2 \mu} \cdot \frac{d\left(B^{2}\right)}{d x}-M \alpha_{i} n_{i} n_{a} V_{i}$

$j=\sigma(E+V \times B)=\frac{1}{\mu} \cdot \frac{d B}{d x}$

where $\alpha_{i}$ is the ionization rate, $\mathrm{P}_{\mathrm{i}}, \mathrm{P}_{\mathrm{e}}$ are ion and electron pressures respectively. We use the equation of state for an ideal gas (i.e. $\mathrm{P}=\mathrm{nkT}$ ). Due to the high electron conductivity, electron temperature variation across the ionization layer is expected to be small and is neglected. It is assumed that electron impact ionization is the dominant process in the ionization layer, while recombination is unimportant. As a first 
approach we consider plasma flow with a uniform current distribution that corresponds to the slow current layer stage of a PPT. This means that we will assume that $j=\sigma\left(E+V_{i} x B\right)=$ const. This assumption simplifies the last equation.

We use the following dimensionless variables: $\xi=\mathrm{x}_{\mathrm{i}} \mathrm{n}_{\mathrm{io}} / \mathrm{C}_{\mathrm{ia}} ; \mathrm{b}=\mathrm{B} / \mathrm{B}_{1} ; \mathrm{n}=\mathrm{n}_{\mathrm{i}} / \mathrm{n}_{\mathrm{io}} ; \mathrm{v}=\mathrm{V}_{\mathrm{i}} / \mathrm{C}_{\mathrm{s}}$., where $\mathrm{C}_{\mathrm{ia}}$ is the heavy particle thermal velocity, $\mathrm{B}_{1}$ is the magnetic field at the edge of the Knudsen layer, and $\mathrm{n}_{\mathrm{io}}$ is the equilibrium plasma density at the edge of the ionization layer.

Taking the above into account, the system is as follows:

$$
\begin{aligned}
& \frac{d b}{d \xi}=-\alpha \\
& \left(v^{2}-1\right) \frac{d v}{d \xi}=-\left(v^{2}+1\right) \varepsilon^{0.5}(1-n)-\frac{v}{2 n} \cdot \beta \frac{d^{2} b}{d \xi^{2}} \\
& v \frac{d n}{d \xi}=-n \frac{d v}{d \xi}+n \varepsilon^{0.5}(1-n)
\end{aligned}
$$

where $\alpha=\mathrm{C}_{\mathrm{ia}} /\left(\mathrm{d} \alpha_{\mathrm{i}} \mathrm{n}_{\mathrm{io}}\right) ; \beta=\left(\mathrm{V}_{\mathrm{a}} / \mathrm{C}_{\mathrm{s}}\right)^{2} ; \varepsilon=\left(\mathrm{T}_{\mathrm{i}}+\mathrm{T}_{\mathrm{e}}\right) / \mathrm{T}_{\mathrm{i}}$

This system of equations can be solved under the following boundary condition: $n=0$. Another additional boundary condition is the velocity at the edge of the ionization layer. It was shown in the previous section that the velocity at the edge of the Knudsen layer can vary in the range from very small $v<<1$ up to the local sonic speed. Note that the velocity presented in Fig. 3 is normalized by the local sound speed determined by the heavy particle temperature at the Knudsen layer edge. This temperature is close to the surface temperature and much smaller than that of the electrons in the ionization layer. Therefore, a physically reasonable condition for the velocity would be $v<<1$.

\section{Small plasma acceleration limit}

Firstly let us consider an asymptotic solution for the case of small plasma acceleration $\left(v^{2}<<1\right)$. In this case, equation 13 is reduced to: 


$$
\frac{d v}{d \xi}=\varepsilon^{0.5}(1-n)+\frac{v}{2 n} \cdot \beta \frac{d^{2} b}{d \xi^{2}}
$$

By substitution of this equation into Eq. 14 we have

$$
\frac{d n}{d \xi}=\alpha \beta(1-\alpha \xi)
$$

The last equation has a solution:

$n=\alpha \beta \xi-0.5 \alpha^{2} \beta \xi^{2}$

One can see that the problem is determined by two parameters $\alpha$ and $\beta$. Therefore, the ionization layer thickness can be determined as solution of this equation for $n=1$ :

$\xi^{*} \approx 1 /(\beta \alpha)$

where $\xi^{*}$ is the thickness of the ionization layer

\section{Plasma density behavior near the sonic transition.}

In this section, we describe the behavior of the plasma density near the sonic transition point. We are looking here for the solution with regular sonic transition. This condition requires that the right hand side of Eq. 13 be zero at the sonic plane. In general, using L'Hopital's rule, one can find the velocity gradient near the sonic plane, which is finite. This procedure was used by a number of authors (see for instance Ref. 18). The numerator on the right hand of Eq. 13 has the form:

$$
0=-\left(v^{2}+1\right) \varepsilon^{0.5}(1-n)-\frac{v}{2 n} \cdot \beta \frac{d^{2} b}{d \xi^{2}}
$$

The equation for the density is: 
$n^{2}-n+\frac{\alpha \beta}{\varepsilon^{0.5}}(1-\alpha \beta)$

Finally the solution that allows regular transition has the following expression for density in the vicinity of the sonic point:

$\left.n=0.5\left(1+\sqrt{1-\frac{\alpha \beta}{\varepsilon^{0.5}}(1-\alpha \beta}\right)\right)$

This is an expression for the density behavior near the sonic point in the case of the regular sonic transition.

\section{Numerical examples}

In this section we describe some numerical examples of the solution of the ionization layer problem (Eqs. 12-14) with three free parameters: $\alpha, \beta$, and $\varepsilon$.

The calculations are shown in Figs. 4, 5. Plasma density and velocity in the ionization layer with $\alpha$ as a parameter are shown in Fig. 4. Parameter $\alpha$ represents an effect of ionization. When $\alpha$ is large this means that the ionization rate is large. One can see that when $\alpha$ is large the ionization layer thickness (determined by condition $n=1)$ becomes smaller. When $\alpha$ is small enough $(\alpha<0.03)$ the ionization equilibrium condition cannot be met. This is shown in the bottom of Fig. 4, where plasma velocity in the ionization layer is shown. It can be seen that the plasma accelerates up to the sonic velocity when $\alpha$ is small before ionization equilibrium can be reached.

Similarly, a dependence on the parameter $\beta$ is found. Parameter $\beta$ represents an effect of the electromagnetic acceleration. When $\beta$ is large the plasma acceleration is small in the subsonic region and as a result, ionization equilibrium can be reached. 
One can conclude that there is a range of parameters when strong ionization can be achieved, i.e. when $\beta>60$ and $\alpha<0.03$. These conditions are necessary conditions for the existence of the ionization layer. An important conclusion that can be derived is that in order to have significant ionization, the ionization and accelerations regions must be separated. It should also be noted that the above numerical results are obtained considering only the subsonic region and therefore a singularity is found. In reality, this singularity disappears if the condition for regular sonic transition is taken into account. In this case, the electron density is calculated according to Eq. 21.

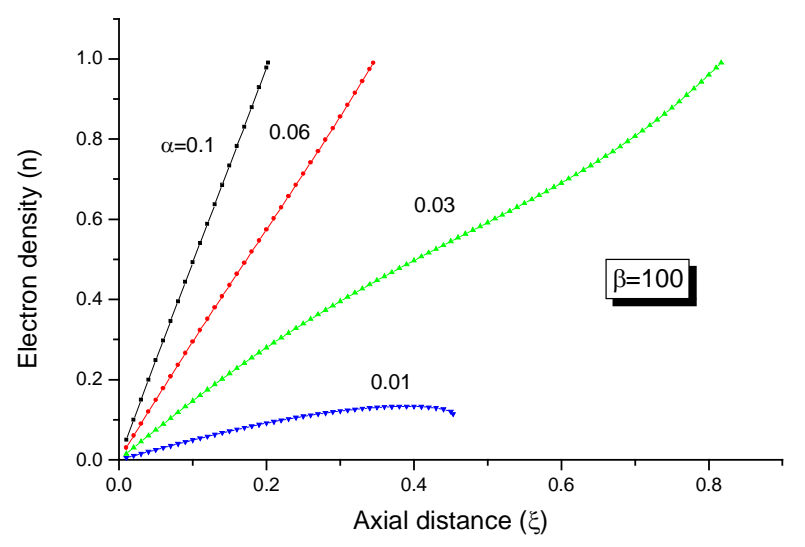

(a)

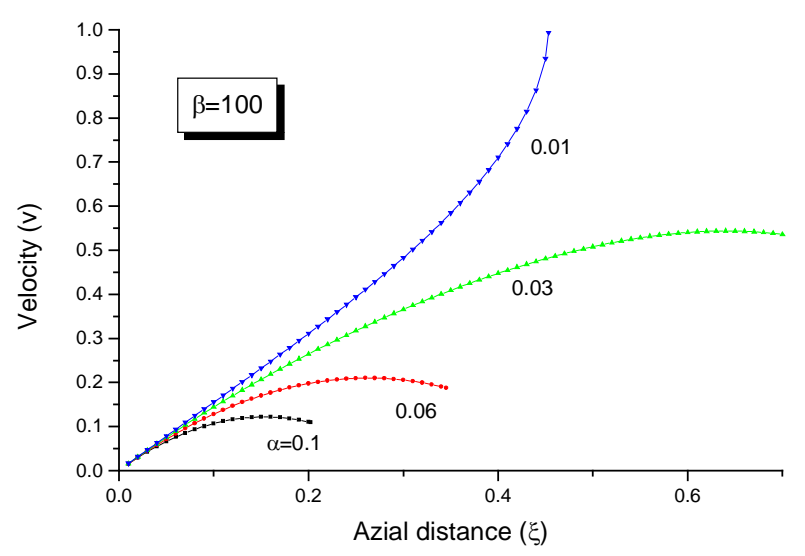

(b)

Figure 4. Plasma and velocity distribution in an ionization layer with $\alpha$ as a parameter 


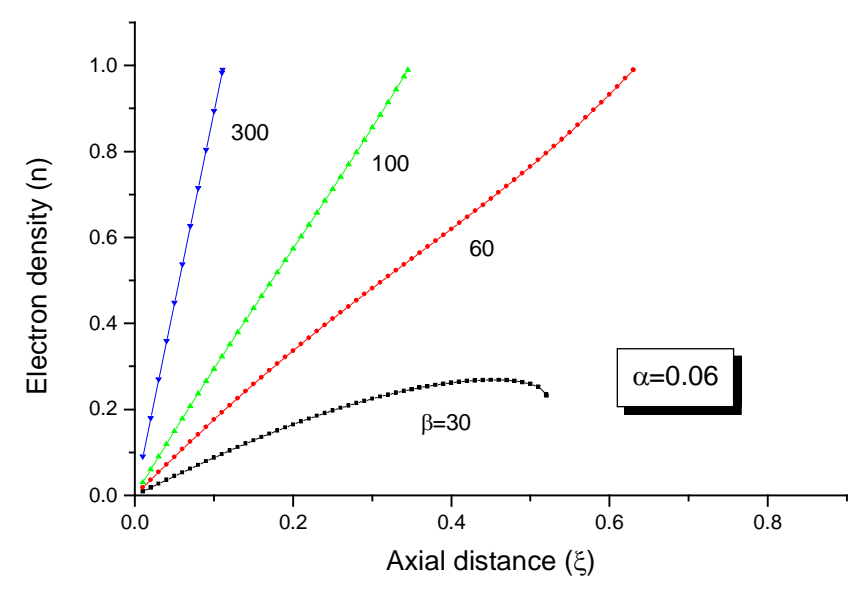

(a)

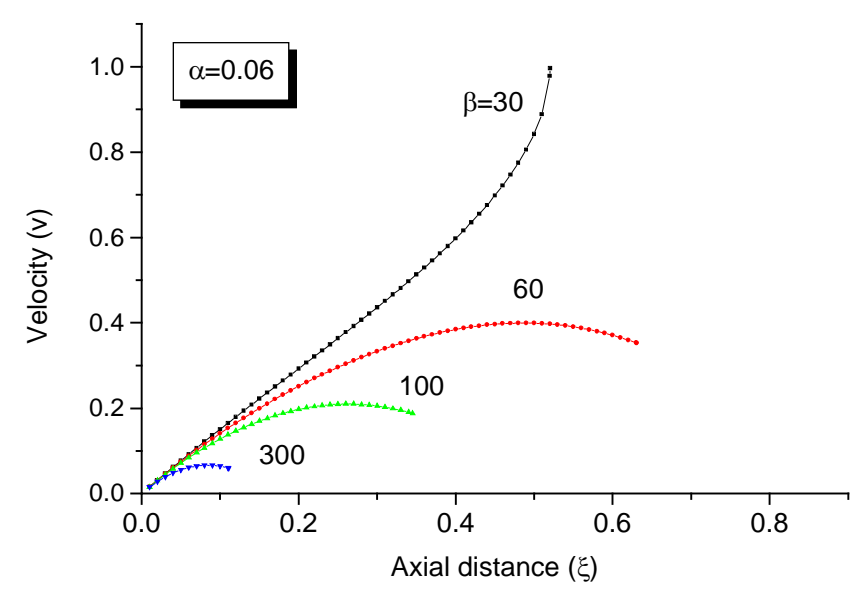

(b)

Figure 5. Plasma and velocity distribution in an ionization layer with $\beta$ as a parameter

In the next section, we apply this model for the specific case of a micro-pulsed plasma thruster, where the driving parameters are determined for a specific situation and also the current density is calculated instead of using a simplified approach. 


\section{Application to the micro-PPT}

In this section we will apply above results to a particular plasma device, micro-pulsed plasma thruster. In order to calculate current density in the near field we assume that the magnetic field has only an azimuthal component and also neglect the displacement current. The geometry of the problem is shown in Fig. 1. The combination of the Maxwell equations and electron momentum conservation gives the following equation for the magnetic field:

$\partial \mathbf{B} / \partial \mathrm{t}=1 /(\sigma \mu) \nabla^{2} \mathbf{B}-\nabla \times(\mathbf{j} \times \mathbf{B} /(\mathrm{eN}))+\nabla \times(\mathbf{V} \times \mathbf{B})$

A scaling analysis shows that the various terms on the right hand side of Eq. 22 may have importance in different regions of the plasma plume and therefore a general end-to-end plasma plume analysis requires keeping all terms in the equation. In the case of the near plume of the micro-PPT with a characteristic scale length of about $1 \mathrm{~cm}$ the magnetic Reynolds number $\operatorname{Re}_{\mathrm{m}}<<1$ and therefore the last term can be neglected. Taking this into account in dimensionless form, Eq. 22 can be written as:

$\operatorname{Re}_{\mathrm{m}} \partial \mathbf{B} / \partial \mathrm{t}=\nabla^{2} \mathbf{B}-(\omega \tau) \cdot\{\nabla \times(\nabla \times \mathbf{B} \times \mathbf{B})\}$

where $(\omega \tau)$ is the Hall parameter that measures the Hall effect. Therefore, depending on the plasma density, the Hall effect may be important for the magnetic field evolution. In addition, our estimations show that the Hall parameter $\omega \tau<<1$ if the plasma density near the Teflon surface $\mathrm{N}>10^{23} \mathrm{~m}^{-3}$. This case is realized in the micro-PPT so the Hall effect is expected to be small for this particular case.

The boundary conditions are similar to that described in Ref. 9. We assume that the current is uniform on both electrodes that allows us to estimate the current density on the cathode $j_{c}$ and on the anode $j_{a}$. The magnetic field is assumed to vary as $1 / \mathrm{r}$ on the upstream boundary. At the lateral boundary we assume that the normal current $\mathrm{j}_{\mathrm{n}}=0$. The downstream boundary is considered to be far enough away that $\mathrm{B}=0$ can be assumed. Along the centerline the magnetic field is zero. 
Having magnetic field distribution we can calculate current density as shown in Fig. 6, where also microPPT geometry is shown for reference. Now in order to calculate ionization layer near the Teflon face we will use current density distribution similar to that shown in Fig. 6 instead of assuming j=const as was done in the previous section.

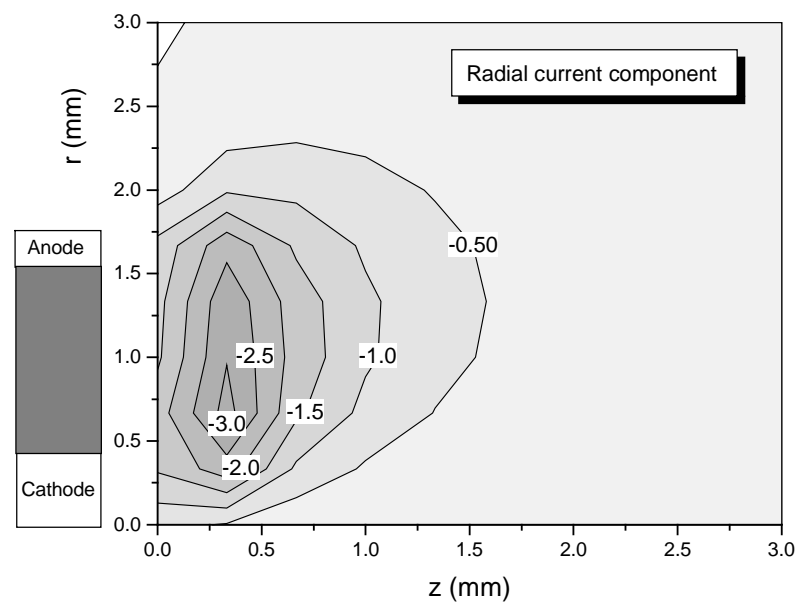

Figure 6. Radial component of current density in the near field of the micro-pulsed plasma thruster

In order to calculate ionization rate for C-F plasma we will use electron impact ionization cross section that is available from elsewhere. ${ }^{19}$ Firstly, we have calculated the ionization layer (according to Eqs. 1214) to study if ionization equilibrium can be achieved. The criteria that was used here is whether ionization equilibrium can be achieved (i.e. $n \rightarrow 1$ ) under considered conditions. The solution depends upon Teflon surface temperature $T_{s}$, electron temperature $T_{e}$ and the current $I$. The specific example that is considered here is the $1 / 4$ " diameter micro-PPT (Ref. $5,11,12$ ). In the $\mathrm{T}_{\mathrm{s}}-\mathrm{T}_{\mathrm{e}}$ plane the curves that separate region with equilibrium are shown in Fig. 7. It can be seen that the separation curve depends upon current. In the region that is below to the curve the plasma conditions allow to establish equilibrium $(n \rightarrow 1)$. For comparison we have calculated $T_{s}-T_{e}$ dependence from simulations according to the plasma generation model of micro-PPT described elsewhere. ${ }^{9,11}$ One can see from comparison results (Figs. 7 a,b) that during discharge there are regimes where equilibrium breaks down. 

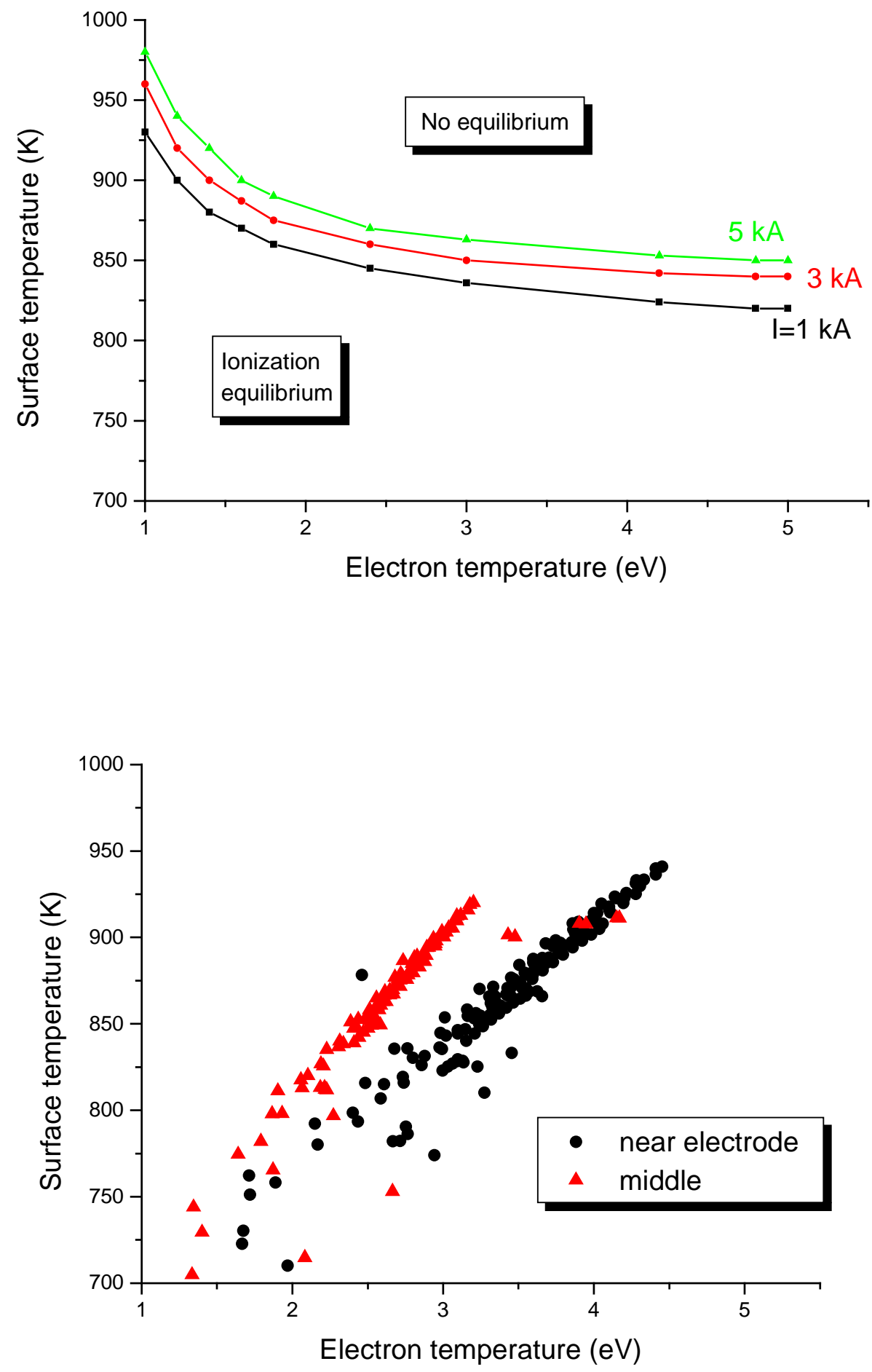

Figure 7. Ts-Te plane. Separation between two regions and calculated dependence of surface temperature and electron temperature. Sampling was taking at two locations, in the midway between electrodes and near outer electrode (see also Fig. 1) 


\section{Comparison with experiment}

In this section we present measured and predicted electron and neutral density distributions in the near field plume for one micro-PPT design. The calculated data correspond to the model with non-equilibrium ionization. In order to calculate plasma composition we used the model presented in Sec. III. We have assumed that regular sonic transition occurs in PPT and therefore electron density behavior near the sonic plane should be according to Eq. 21. It was assumed that at the beginning of the ionization layer plasma temperature is different from the temperature in the plasma bulk. The calculation here presented for the ratio of the electron temperature at the beginning of the ionization layer to the bulk electron temperature $\theta$ as a parameter. In future work we intend to determine this free parameter.

The plasma plume expansion was calculated using previously developed hybrid PIC-DSMC model. ${ }^{7-9}$ In this model, the neutrals and ions are modeled as particles while electrons are treated as a fluid. Elastic (momentum transfer) and non-elastic (charge exchange) collisions are included in the model. The grids employed in this computation are also similar to those used previously (Ref.7). The particle collisions are calculated using the direct simulation Monte Carlo (DSMC) method ${ }^{20}$. Momentum exchange cross sections use the model of Dalgarno et al. ${ }^{21}$, while charge exchange processes use the cross sections proposed by Sakabe and Izawa ${ }^{22}$. Acceleration of the charged particles is computed using the Particle-InCell method (PIC) ${ }^{23}$.

An experimental data presented here was obtained using a Herriott Cell interferometer. Electron and neutral density measurements are taken on a $6.35 \mathrm{~mm}$ (1/4") diameter MicroPPT at AFRL. Details about measurement technique and more experimental results can be found elsewhere. ${ }^{12,}{ }^{24}$ Figure 8 shows the experimental data co-plotted with model predictions. Plasma density peaks at about $20 \times 10^{15} \mathrm{~cm}^{-3}$ while 
neutral density peaks at about $200 \times 10^{15} \mathrm{~cm}^{-3}$ and decreases by several orders of magnitude towards the pulse end. The experimental data was taken at a discharge energy of $7.5 \mathrm{~J}$. There is uncertainty in the measurements. First of all they are due to shot-to-shot variations in thruster firing. There are uncertainties related to the spatial resolution. More detail about accuracy of these measurements can be found in Refs. $12,24$.

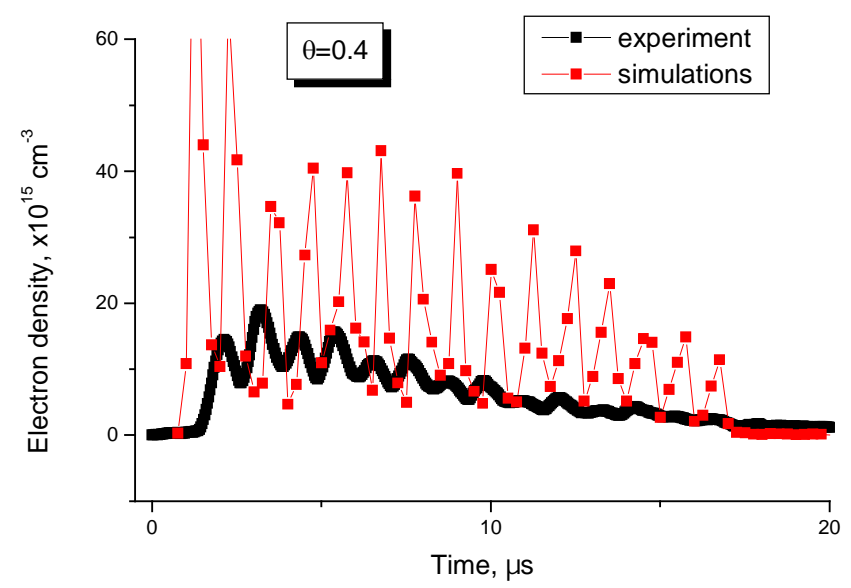

(a)

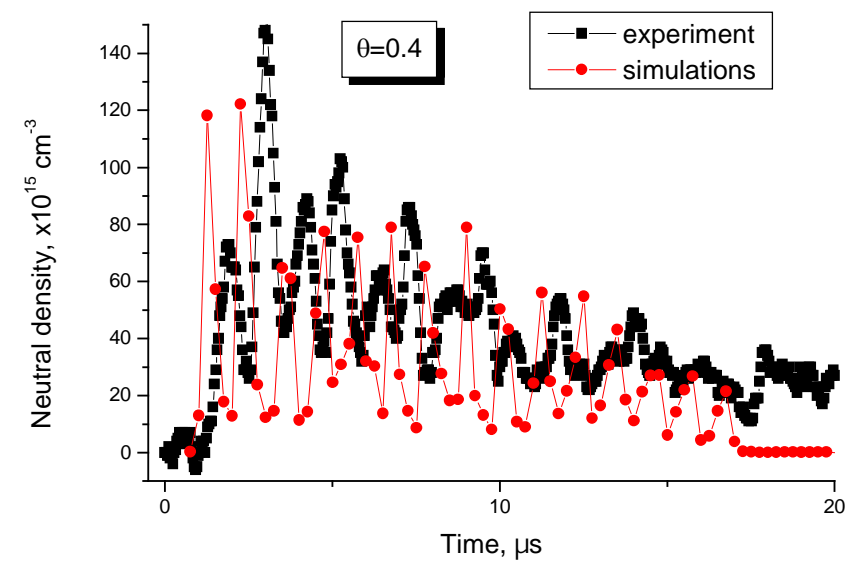

(b)

Figure 8. Electron and neutral density temporal behavior. Comparison with experiment. Experimental data were taken from Ref. 12. 


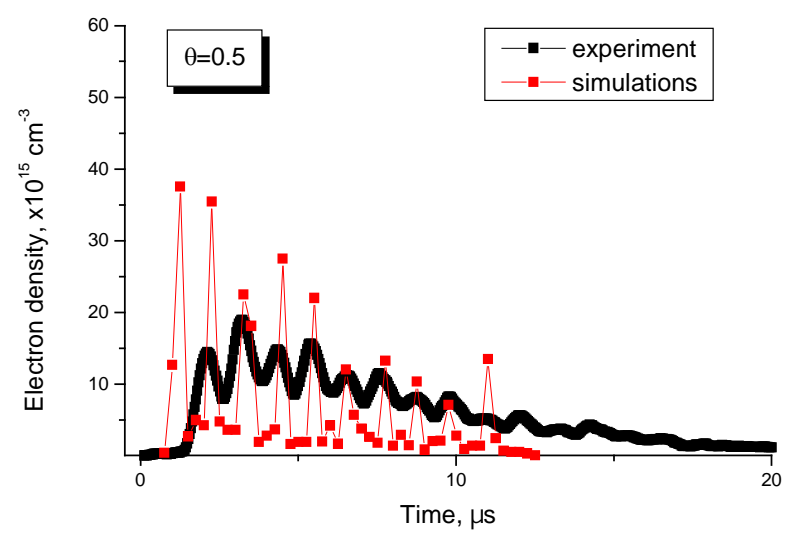

(a)

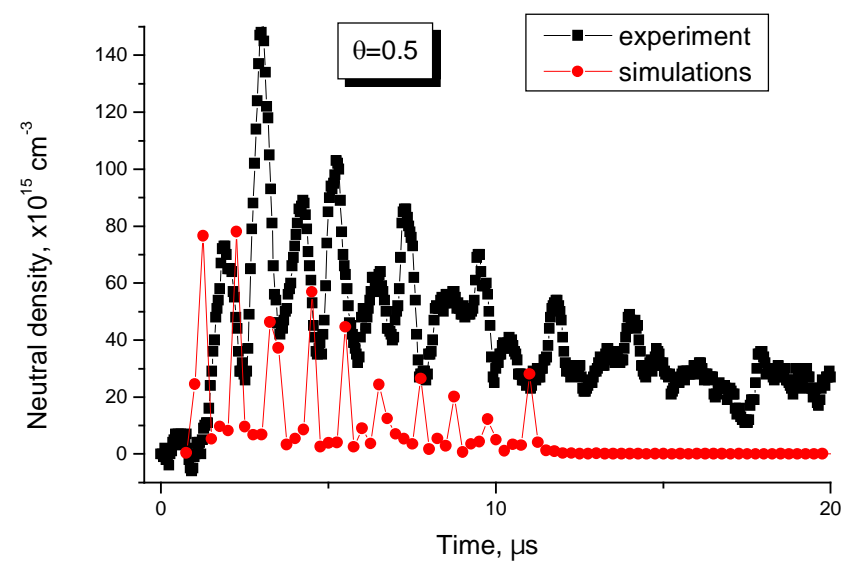

(b)

Figure 9. Electron and neutral density temporal behavior. Comparison with experiment. Experimental data were taken from Ref. 12.

A comparison of the simulation and experimental results are shown in Fig. 8 and Fig. 9 with $\theta$ as a parameter. One can see that the model generally satisfactory predicts both the plasma density level and temporal behavior during the entire pulse. In the case of $\theta=0.4$ the model overpredicts electron density but gives very good agreement on the neutral density. When parameter $\theta$ increases both electron and neutral densities decreases as shown in Fig. 9. The physical meaning of this parameter is that there is electron 
temperature gradient from the plasma bulk to the propellant surface. In this model the ratio of the electron temperature at the beginning of the ionization layer to the bulk electron temperature $\theta$ is used as a parameter. In future work we intend to determine this free parameter.

\section{Summary}

In this paper we report about progress in micro-PPT plasma modeling with the main empathies on the plasma-surface interactions. Two inter-related problems are considered here, namely ablation and formation of the ionization layer. We found that there is a smooth transition between two ablation modes with small velocity at the edge of the Knudsen layer and limited (sound) velocity at the Knudsen layer edge. In the first case fraction of backflux is very large, while in the second case it about $18 \%$ of the

primary flux. It was concluded that the transition between these two modes occurs as current density increases. The model of the non-equilibrium ionization is developed. Specific example of $1 / 4$ " micro-PPT fired at $7.5 \mathrm{~J}$ is considered. It is shown that both equilibrium and non-equilibrium ionization regimes can be realized in PPT. Based on non-equilibrium ionization model plasma generation and near-plume expansion was calculated for micro-PPT. Generally good agreement with experimental measurements of the electron and neutral densities is found.

\section{Acknowledgements}

The authors gratefully acknowledge financial support by the Air Force Office of Scientific Research through grant F49620-02-1-0084 and by the Air Force Research Laboratory. We also acknowledge Dr. Greg. G. Spanjers, Erik L. Antonsen, Prof. R.L. Burton and Prof. I.I. Beilis for numerous and valuable discussions. 


\section{REFERENCES}

${ }^{1}$ R. L. Burton and P. J. Turchi, "Pulsed plasma thruster", Journal of Propulsion and Power, Vol.14, No. 5, 1998, pp. 716-735.

${ }^{2}$ R.J. Vondra and K.I. Thomassen, "Flight qualified pulsed plasma thruster for satellite control", Journal of Spacecraft and Rockets, Vol. 11, No. 9, 1974, pp. 613-617.

${ }^{3}$ P. J. Turchi, Directions for improving PPT performance, Proceeding of the $25^{\text {th }}$ International Electric Propulsion Conference, vol. 1, Worthington, OH, 1998, pp. 251-258.

${ }^{4}$ R. A. Spores, R. B. Cohen and M. Birkan, “The USAF Electric propulsion program”, Proceeding of the $25^{\text {th }}$ International Electric Propulsion Conference, vol. 1, Worthington, OH, 1998, 1997, p.1.

5 Spanjers, G.G., White, D., Schilling, J., Bushman, S., Lake, J., Dulligan, M., “AFRL MicroPPT Development for the TechSat21 Flight,” $27^{\text {th }}$ Intl Electric Propulsion Conference, IEPC paper 2001-166, Pasadena, CA 2001.

${ }^{6}$ N. A. Gatsonis and X. Yin, Axisymmetric DSMC/PIC simulation of quasineutral partially ionized jets, AIAA paper $97-2535,1997$

${ }^{7}$ I. D. Boyd, M. Keidar, and W. McKeon, Modeling of a pulsed plasma thruster from plasma generation to plume far field, Journal of Spacecraft and Rockets, Vol. 37, No. 3, 2000.

${ }^{8}$ M. Keidar and I.D. Boyd, "Device and plume model of an electrothermal pulsed plasma thruster", Paper AIAA2000-3430.

${ }^{9}$ M. Keidar and I.D. Boyd, "Electromagnetic effects in the near field plume exhaust of a pulsed plasma thruster", AIAA Paper 2001-3638

${ }^{10}$ Gulczinski, F., Dulligan, M., Lake, J., and Spanjers, G.G., :Micropropulsion Research at AFRL," Paper AIAA2000-3255.

${ }^{11}$ M. Keidar, I. D. Boyd, F. S. Gulczinski, E. L. Antonsen, and G. G. Spanjers, "Analyses of Teflon Surface Charring and Near Field Plume of a Micro-Pulsed Plasma Thruster," IEPC Paper 01-155, October 2001.

${ }^{12}$ E. L. Antonsen, R.L. Burton, and G.G. Spanjers, "High resolution laser diagnostics in millimeter-scale micro Pulsed Plasma Thruster", IEPC Paper 01-157, October 2001

${ }^{13}$ M. Keidar, I.D. Boyd and I.I. Beilis, "On the model of Teflon ablation in an ablation-controlled discharge", J. Phys. D: Appl. Phys., 34, 2001, pp. 1675-1677.

${ }^{14}$ M. Keidar, J. Fan, I.D. Boyd and I.I. Beilis, "Vaporization of heated materials into discharge plasmas", J. Appl. Phys., 89, 2001, pp. 3095-3098.

${ }^{15}$ I. Mikellides, Ph.D Thesis, Ohio State University, Columbus 2000

${ }^{16}$ P.J. Turchi et. al., "Pulsed plasma thruster for microsatellite propulsion: techniques for optimization", in book "Micropropulsion for Small Spacecraft", Michael M. Micci and Andrew D. Ketsdever, Editors, Progress in Astronautics and Aeronautics, Volume 187, AIAA, 2000. 
${ }^{17}$ R.L. Burton et al., Pulsed plasma thruster performance for microspacecraft propulsion, in book "Micropropulsion for Small Spacecraft”, Michael M. Micci and Andrew D. Ketsdever, Editors, Progress in Astronautics and Aeronautics, Volume 187, AIAA, 2000.

${ }^{18}$ A. Fruchtman, N.J. Fisch, and Y. Raitses, Phys. Plasmas, 8, 1048, 2001

${ }^{19}$ IAEA Aladin database

${ }^{20}$ G.A. Bird, "Molecular gas dynamics and the direct simulation of gas flows" (Clarendon Press, Oxford, 1994).

${ }^{21}$ A. Dalgarno, M.R.C. McDowell and A. Williams, The modilities of ions in unlike gases, Proc. Of Royal Soc. Of London, Vol. 250, April 1958, pp. 411-425.

${ }^{22}$ S. Sakabe and Y. Izawa, Simple formula for the cross sections of resonant charge transfer between atoms and their ions at low impact velocity, Physical Rev. A: General Physics, v. 45, No. 3, 1992, pp. 2086-2089.

${ }^{23}$ C.K. Birdsall and A.B. Langdon, Plasma Physics via Computer Simulation, Adam Hilger Press, 1991.

${ }^{24}$ E.L. Antonsen, Herriott Cell Interferometry for Pulsed Plasma Density Measurements, MS Thesis, University of Illinois at Urbana-Champaign, 2001 